\title{
The Thermodynamics of Relativistic Multifluid Systems
}

\author{
Bob Osano 1,2 \\ ${ }^{1}$ Centre for Higher Education Development, \\ University of Cape Town (UCT), Rondebosch 7701, Cape Town, South Africa \\ ${ }^{2}$ Cosmology and Gravity Group, Department of Mathematics and Applied Mathematics, \\ University of Cape Town (UCT), Rondebosch 7701, Cape Town, South Africa
}

\begin{abstract}
This article extends the single-fluid relativistic irreversible thermodynamics theory of Müller, Israel, and Stewart (hereafter the MIS theory) to a multifluid system with inherent species interactions. This is illustrated in a two-fluid toy model where an effective complex 4-velocity plays the role of a primary dynamical parameter. We find that an observer who resides in the real part of this universe will notice that their knowledge of the universe parametrized using real, rather than imaginary, quantities is insufficient to fully determine properties such as the total energy density, pressure, or entropy. In fact, such an observer will deduce the existence of some negative energy that affects the expansion of their perceived real universe.
\end{abstract}

Keywords: Relativistic, Thermodynamics, Particles, Single-Fluid, Multi-Fluid, Cosmology

DOI: $10.31526 /$ lhep.2020.141

\section{INTRODUCTION}

The extended relativistic thermodynamics theory by Müller, Israel, and Stewart developed in $[1,2,3,4]$ has found wide application in scenarios where the material content under investigation can be modelled using single-fluid approximation, whether the material is made of (1) one species or (2) several species whose properties are given by the average or the bulk behaviour. This treatment forms the foundation of most studies in relativistic nonequilibrium thermodynamics found in the literature for idealised fluids. How closely this approximation models realistic fluid mixture needs to be investigated. To this end, we formulate a multifluid approximation as the first step in the relaxation of the conditions governing the MIS theory. We will demand that MIS theory is recovered in the appropriately motivated limit. Part of the requirement will be that the resulting system of differential equations must retain their hyperbolicity $[5,4]$ in line with the principle of causality and be stable [6] for the formulation to be viable and predictive [7]. The aim of this article is modest and is primarily to present a formalism for the thermodynamics of the relativistic system of multifluid. Since we intend to extend the MIS theory, we will begin with the assumption that the MIS model is the standard theory and extension must necessarily recover it when subjected to physically motivated constraints or conditions that impact the nature of fluid approximation. Several properties that are established in the single-fluid approximation suddenly lose clarity. In terms of thermodynamics, these are the definitions of (i) a universal temperature [8,9], (ii) entropy, (iii) heat, and (iv) work, to mention but the primary one. This lack of clarity impacts the laws of thermodynamics and will require scrutiny.

The problem of defining a universal temperature was first encountered in the nonequilibrium thermodynamics theories in the single-fluid approximation. At the core of the problem is the seeming nonexistence of a Lorentzian type transformation between reference frames that readily recover the black-body temperature given the necessary constraints [8]. Some progress has been made in this regard, see [9], for example, but the debate is not closed. It is important to emphasise that we will make some assumptions in this paper regarding how temperature is transformed to allow for progress but the description should not be taken as definitive. A detailed analysis of such transformations will be pursued in the future [10]. As for the definition of entropy, it is known that when solving gravitational field equations, the standard approach takes into account the bulk effects and ignores the surface effects. But it is known that when surface terms are evaluated at the horizon, they give the entropy of such a horizon (see [11] and references therein). This suggests that entropy should ideally include the body and surface terms.

The characterization of heat and work is also less straight forward; see [12]. It is known that heating, although heat is defined, of a space-time that is endued with a certain microscopic degree of freedom and which is capable of exhibiting thermal phenomena will necessarily create microstructures. It is sensible to consider the converse of this and, therefore, ask how microstructures affect the macroproperties in space-time dynamics. It is this notion of microstructures that motivates the formulation that we develop in this article.

This article is organised as follows. Section 2 discusses the thermodynamics of single-fluid approximation while Section 3 discusses the thermodynamics of multifluid approximation. Section 4 discusses the special case of two-fluid approximation. Section 5 gives the discussion and the conclusion.

\section{IRREVERSIBLE THERMODYNAMICS AND THE SINGLE-FLUID APPROXIMA- TION}

In order to understand the complicated nature of multifluid dynamics, it is useful to review the single-fluid approximation in the context of general relativity and cosmology. Early theories on single-fluid irreversible thermodynamics $[13,14,15$, $16,17,18,19,20,21]$ were plagued by the pathology that they predicted instantaneous propagation of viscous and thermal effects, given the parabolic nature of the resultant differential equations. This made such theories to be predictive only for slowly varying systems. The pathology was traced, see [1], to the nonperturbative [10] truncation procedure which led to the 
dropping of quadratic terms from the heat and viscous stresses in the expression for the entropy 4 -vector. The entropy, in this case, includes both the material and 4-momentum fluxes. This was clearly not suitable for fast varying systems and a new theory was, therefore, required. This led to the development of the theory that we will discuss next.

\subsection{The Müller-Israel-Stewart (MIS) Theory}

Also called Israel-Stewart theory, the MIS theory is a theory for relativistic irreversible thermodynamics based on singlefluid approximation. The state of the fluid is generally given by three entities: the stress-energy-momentum tensor $T^{\mu v}$, the particle flux $N^{\mu}$, and the entropy flux $S^{\mu}$. The momentum tensor and the particle flux obey their respective conservation laws, $T^{\mu \nu}{ }_{; \nu}=0$ and $N^{\mu}{ }_{; \mu}=0$, while the entropy vector obeys the second law of thermodynamics $S^{\mu} ; \mu>0$. The semicolon denotes the covariant derivative. If $u^{a}$ is a time-like vector and $h_{\mu \beta}$ is an orthogonal projection tensor, it follows that the energy density $\rho=T^{\mu v} u_{\mu} u_{v}>0$. $T^{\mu v}$ has a time-like unit vector $u_{\left.\right|_{E}}^{\mu}$ (i.e., $u_{\left.\right|_{E}}^{\mu} u_{\mu_{\left.\right|_{E}}}=-1$ ). This is not the only time-like unit vector that one can find for such a fluid. In fact, one can define other unit vectors; for example, $u_{\left.\right|_{N}}^{\mu}=N^{\mu} / \sqrt{-N^{\mu} N_{\mu}}$ or even $u_{\left.\right|_{S}}^{\mu}=S^{\mu} / \sqrt{-S^{\mu} S_{\mu}}$. It follows that $u_{\left.\right|_{N}}^{\mu}=u_{\left.\right|_{E}}^{\mu}$ for fluid at equilibrium, which suggests that there exists a unique time-like 4 -velocity vector which will be denoted by $u^{\mu}$. The full set of relevant equations for the perfect fluid case, synonymous with the thermodynamic equilibrium, take

$$
\begin{aligned}
T^{\mu v} & =\rho u^{\mu} u^{v}+p h^{\mu v}, \\
N^{\mu} & =n u^{\mu}, \\
S^{\mu} & =s u^{\mu},
\end{aligned}
$$

where $p=T^{\mu v} h_{\mu v}$ is the isotropic pressure, $\rho$ is again the energy density, $n$ is the number density, and $s$ is the flux density. An alternative formulation, in terms of divergence type functions, is given in the appendix (Appendix A). In the present formulation, the equation of state is given by $p=p(\rho, s)$.

Deviation from equilibrium can then be characterised using

$$
u_{\left.\right|_{N}}^{\mu}-u_{\left.\right|_{E}}^{\mu}=\mathcal{V}^{\mu} \neq 0,
$$

where the requirement that $\mathcal{V}^{\mu}<<1$ may be used to characterise the close to equilibrium or quasi-equilibrium [22]. In this case, one can define an orthogonal projection tensor $h_{\mu \beta}=$ $g_{\mu \beta}+u_{\mu} u_{\beta}$. This is because of

$$
\begin{aligned}
u_{\left.\right|_{N}}^{\mu} u_{\left.\mu\right|_{N}} & =\mathcal{V}^{\mu} \mathcal{V}_{\mu}+\mathcal{V}^{\mu} u_{\left.\mu\right|_{N}}+u_{\left.\right|_{N}}^{\mu} \mathcal{V}_{\mu}+u_{\left.\right|_{E}}^{\mu} u_{\left.\mu\right|_{E}} \\
& =-1
\end{aligned}
$$

when evaluated in the reference frame of a perfect fluid. Since

$$
u_{\left.\right|_{N}}^{\mu}=u_{\left.\right|_{E}}^{\mu}+\mathcal{V}^{\mu}
$$

$\mathcal{V}^{\mu}$ can be thought of a linear perturbation. $\mathcal{V}^{\mu} \mathcal{V}_{\mu}$ is a product of first-order perturbation terms and therefore a higher-order perturbation. Similarly, $\mathcal{V}^{\mu} u_{\mu_{N}}$ is a first order. These terms are dropped when evaluated in the frame. As in the case of perfect fluids, we do not have a unique 4-velocity vector for any arbitrary nonperfect fluid. A thermodynamic formulation for such nonprefect fluid must necessarily incorporate anisotropic stress and where appropriate heat exchange. These are characterised using the quantities $\pi_{\mu v}$, the anisotropic stress tensor, and $q_{\mu}$, the energy flux vector, respectively.

An appropriate starting point in the formulation of a theory of irreversible thermodynamics is to allow key parameters such as entropy and energy-momentum tensor to be functions of a broader number of properties, over above the standard volume and internal energy. To formalise such extension, let these properties be given by the generic scalar $f$, vector $f^{\mu}$, and tensor $f^{\mu \nu}$, in which case $s=s\left(f, f^{\mu}, f^{\mu \nu}\right)$. These tensors of ranks 0 , 1 , and 2 can be explicitly defined to have the physical meaning as discussed in $[22,7,23]$. We note here that the tensors denote both bulk and surface terms. This is important as it will allow our description to include surface entropy. It follows that

$$
d s=\frac{\partial s}{\partial f} d f+\frac{\partial s}{\partial f^{\mu}} d f^{\mu}+\frac{\partial s}{\partial f^{\mu \nu}} d f^{\mu \nu} .
$$

In general, the Gibbs relation is often expressed in terms of tensors of rank 0 and rank 1. Equation (6) introduces the dependency on a rank 2 tensor. This generalized expression is clearly an extension of the Gibbs relation. We note that we could have more than one of these intrinsic properties characterized by a scalar, a vector, or a tensor. For example, the internal energy and volume are both scalars while heat is a vector. The coefficients in Equation (6) can then be treated in the usual manner with the case of $\partial s / \partial E=1 / T$, where $T=T\left(f, f^{\mu}, f^{\mu \nu}\right)$ is a nonlinear temperature. It suffices to say that it is easy to recover the standard Gibbs relation by restricting Equation (6) to scalars. For the standard set of properties, it can be shown that

$$
S^{\mu}=s u^{\mu}+\frac{1}{T} q^{\mu}-\mathcal{Q}^{\mu}
$$

where $\mathcal{Q}$ denotes a collection of second-order terms and takes the form

$$
\begin{aligned}
\mathcal{Q}^{\mu}= & \frac{u^{\mu}}{2 T}\left[\beta_{0} \Pi^{2}+\beta_{1} q_{\mu} q^{\mu}+\beta_{2} \pi^{\mu v} \pi_{\mu v}\right] \\
& -\frac{1}{T}\left(\alpha_{0} \Pi q^{\mu}-\alpha_{1} \pi^{\mu v} q_{v}+\mathcal{F}\right),
\end{aligned}
$$

where $\mathcal{F}$ is a function of energy density, isotropic pressure, energy flux, and the symmetric shear tensor. For a detailed discussion of this term, the coefficients $\beta_{0}, \beta_{1}, \beta_{2}, \alpha_{0}$, and $\alpha_{1}$, and those embedded in $\mathcal{F}$, the reader is referred to [1,2] and for a pedagogical presentation to [22]. It follows that stress-energy momentum tensor for such nonperfect fluid takes the form

$$
T^{\mu v}=\rho u^{\mu} u^{v}+p h^{\mu v}+2 q^{(\mu} u^{v)}+\pi^{\mu v}
$$

and is the Müller-Israel-Stewart theory [3, 1, 2]. The particles flux also has a contribution from possible particle drift, $N^{\mu}=$ $n u^{\mu}+n^{\mu}$, where $n^{\mu}$ is the particle drift in the frame defined by $u^{\mu}$. Two special frames in which the fluid dynamics has a physical meaning, but not the only ones, are the Landau-Liftshitz (energy) frame defined by $u^{\mu}=u_{\left.\right|_{E}}^{\mu} \Rightarrow q^{\mu}=0$ and the Eckart (particle) frame defined by $u^{\mu}=u_{\left.\right|_{N}}^{\mu} \Rightarrow n^{\mu}=0$. These are related via Equation (4). The existence of different frames, in single-fluid approximation, implies the existence of different projection tensors whose generic form is $h_{\mu v}=g_{\mu v}+u_{\mu} u_{v}$. The frames of reference increase when one moves from singlefluid and multifluid approximation. Each constituent fluid will 
have a unique energy frame and a particle frame. We would now like to broaden this discussion by considering multiple fluids, where the above generalizations do not readily apply. We expect that there exist some limiting conditions that should allow us to recover the standard MIS theory. The reader will remember that our standard concepts of heat and work are often frame-dependent. This means that the choice of a frame will determine our notion of these two concepts. It has been demonstrated [12] that even the concept of volume may be framedependent leading to disparities in the estimation of fundamental quantities. This lack of clarity becomes even more pronounced when one considers more than one fluid, but as we will show, one can develop global parameters that are linked to the local frame and which allow for ease of physical interpretation. This is pursued in the next section.

\section{IRREVERSIBLE THERMODYNAMICS AND THE MULTIFLUID APPROXIMA- TION}

To make the approximation procedure that we will develop more explicit, it is helpful to borrow the language of fluid dynamics. In this regard, we need to discuss the scales in which fundamental changes take place when fluids mix and how these relate to the modelling techniques used. We adopt the language in [24]. It is known that when different fluids come into contact during their flows, the resultant mixture is inhomogeneous. However, the dynamical act of mixing is a transport process involving temperature, species, and phases that lead to a reduction of inhomogeneity. In an ordinary fluid, the mixing may generate other effects such as reaction or even changes in fluid properties. Mixing is often categorised as macro, meso, and micro. While macromixing is governed by the largest scale of the fluid motion, micromixing is governed by the opposite end of the scale, the smallest scale of the fluid motion and even the molecular motion. In conventional macroscale mixing, the smallest scale of fluid motion is the size of turbulent eddies, also called the Kolmogorov scale. A scale of mixing that lies between macro and micro is referred to as mesomixing. A theory of extended irreversible thermodynamics for single-fluid approximation based on the mesoscale was presented in [25]. Taking some of these properties as analogues of cosmological fluids, we may want to identify a scale reminiscent to the Kolmogorov scale, which we will refer to as the cosmological Kolmogorov scale which would be a cut-off for approximating relativistic fluid properties. In this regard, we will not attempt to investigate relativistic turbulence but instead develop a formalism for two interacting fluids. Assume that the two fluids are denoted by $X$ and $Y$. The dynamical variables are the stress-energy tensors denoted by $T_{\left.\right|_{X}}^{\mu \nu}$ and $T_{\left.\right|_{Y}}^{\mu \nu}$, the particle fluxes denoted by $N_{\left.\right|_{X}}^{\mu}$ and $N_{\left.\right|_{\gamma^{\prime}}}^{\mu}$, and the entropy fluxes denoted by $S_{\left.\right|_{X}}^{\mu}$ and $S_{\left.\right|_{Y}}^{\mu}$. To understand the conservation properties in a multifluid environment, it is important to distinguish between species interactions with dissipative properties (i) without entrainment and (ii) with entrainment. In the first case, individual species obey own conservation laws and cumulative conservation; i.e., if $f_{X}^{\mu v}$ and $f_{Y}^{\mu v}$ are tensor properties for the two species, then $\nabla_{\mu}\left(f_{X}^{\mu v}+f_{Y}^{\mu v}\right)=0$ which may be taken to imply $\nabla_{\mu} f_{X}^{\mu v}=0=\nabla_{\mu} f_{Y}^{\mu v}$. The case involving entrainment is more nuanced and $\nabla_{\mu}\left(f_{X}^{\mu v}+f_{Y}^{\mu v}\right) \neq 0$. In fact, $\nabla_{\mu}\left(f_{X}^{\mu v}+f_{Y}^{\mu v}+f_{X Y}^{\mu v}\right)=0$, where the last terms en- code reaction and other nonstandard interaction properties. We must consider the interaction components $\left(N_{\left.\right|_{X Y} ^{\mu}}^{\mu}, T_{\left.\right|_{X Y}}^{\mu \nu}\right.$, and $\left.S_{\left.\right|_{X Y} ^{\mu}}^{\mu}\right)$ in the multifluid formulation. Hence,

$$
\begin{aligned}
& \nabla_{\mu} \sum_{i} N_{\left.\right|_{i}}^{\mu v}=0=\nabla_{\mu} \sum_{i} T_{\left.\right|_{i}}^{\mu v}, \\
& \nabla_{\mu} \sum_{i} S_{\left.\right|_{i}}^{\mu}>0,
\end{aligned}
$$

where $i=X, Y$ or $X Y$. The $X Y$ incorporates entrainment, where the interaction allows for it $[26,7]$. It is instructive to note that two observers moving with the 4-velocities $u_{\left.\right|_{X}}^{\mu}(=$ $\left.N_{\left.\right|_{X}}^{\mu} / \sqrt{-N^{\mu}{ }_{\left.\right|_{X}} N_{\left.\mu\right|_{X}}}\right)$ and $u_{\left.\right|_{Y}}^{\mu}\left(=N_{\left.\right|_{Y}}^{\mu} / \sqrt{-\left.N^{\mu}\right|_{Y} N_{\left.\mu\right|_{Y}}}\right)$ will have different rest-frames and different projections on their respective frames. These may be denoted by $h_{\left.\right|_{X}}^{\mu v}=g_{\left.\right|_{X}}^{\mu v}+u_{\left.\right|_{X}}^{\mu} u_{\left.\right|_{X}}^{v}$ and $h_{\left.\right|_{Y}}^{\mu v}=g_{\left.\right|_{Y}}^{\mu v}+u_{\left.\right|_{Y}}^{\mu} u_{\left.\right|_{Y}}^{v}$, with the special case $g_{\left.\right|_{X}}^{\mu v} \equiv g_{\left.\right|_{Y}}^{\mu v}$ (this is reminiscent of the energy and the particle frames occupying space with the same geometry). Although we have presented these as projections onto hypersurfaces, they need not be so. Projection tensors could be surface-forming, such as the familiar case in general relativity which allows the curvature to be decomposed into equations that include the Gauss-Codazzi equations or the Gauss-Weingarten relations linking embedded geometry connections to the hypersurface geometry connections. None surface-forming projections also exist and allow for the definition of fluid properties such as shear and vorticity and the familiar Raychaudhuri equations [27, 28]. An alternative way to look at this is to consider one of the fluids, for example, that with the 4 -velocity $u_{\left.\right|_{X}}^{\mu}$. The condition $u_{\left.\right|_{X}}^{\mu} u_{\mu_{X}}=-1$ suggests the existence of the projection tensor $U_{a b \mid X}=-u_{\mu_{\mid X}} u_{\left.v\right|_{X}}$ that obeys the condition $U^{\mu}{ }_{\gamma \mid X} U^{\gamma}{ }_{v \mid X}=U^{\mu}{ }_{v \mid X} \cdot U^{\mu}{ }_{v \mid X}$ projects onto the tangent space of this fluid world-line. We demand that energy and the particle frame of the unified approach satisfy $\left|\hat{u}^{\mu}-\hat{u}_{E}^{\mu}\right|<<1$. Let us now define a resultant 4-velocity $\hat{u}^{\mu}=f\left(u_{\left.\right|_{X}}^{\mu}, u_{\left.\right|_{Y}}^{\mu}\right)$ and the corresponding projection tensor

$$
\hat{h}^{\mu}{ }_{v}=\hat{g}^{\mu}{ }_{v}+\hat{u}^{\mu} \hat{u}_{v}
$$

which projects onto the rest-frame of the fluid mixture such that $\hat{h}_{\mu}^{v} \hat{u}_{v}=0$. We will assume that this observer, $\hat{u}^{\mu}$, is not accelerated in contrast to that considered in [7]. These velocity fields are chosen in such a way that they satisfy the concavity requirement [3]. Once the $\hat{u}^{\mu}$ is chosen, the observer moving with this velocity will record the energy density $\hat{\rho}$ and the particle flux $\hat{N}^{\alpha}=f\left(N^{\mu}{ }_{\mid X}, N^{\mu}{ }_{\mid Y}\right)$, where $f$ denotes 'f unction of .

It follows that the total stress-energy momentum tensor is given by

$$
T^{\mu v}=\sum_{i} T^{\mu v}{ }_{\mid i}
$$

where again $i=X, Y, X Y$. These can be decomposed into the energy density, pressure (isotropic and anisotropic) terms, and the heat term in the standard way and takes the following form:

$$
T_{\mid i}^{\mu v}=\hat{\rho}_{\mid i} \hat{u}_{\mid i}^{\mu} \hat{u}_{\mid i}^{v}+\hat{p}_{\mid i} \hat{h}_{\mid i}^{\mu, v}+2 \hat{u}_{\mid i}^{(\mu} \hat{q}_{\mid i}^{v)}+\hat{\pi}_{\mid i}^{\mu \nu},
$$

where the heat flux vector is given by $\hat{q}_{\mu \mid i}=-\hat{h}_{\mu v \mid i} \hat{u}_{\gamma \mid i} T_{\mid i}^{v \gamma}$ while the anisotropic stress-energy tensor is given by $\hat{\pi}_{\mu v \mid i}=$ $T_{\mid i}^{\gamma \delta}\left(\hat{h}_{\gamma\langle\mu} \hat{h}_{v\rangle \delta}\right)_{\mid i}$. The total entropy also takes the form

$$
S^{\mu}=\sum_{i} S_{\mid i}^{\mu}+S_{\text {surf }},
$$


where again the individual contribution can be expressed in terms of heat vector, temperature, and rest frame defined entropy as will be shown in Equation (31). $S_{\text {surf }}$ represents the entropy enveloping the volume occupied by the two fluids. The generic nature of the formalism presented in this section conceals its significance. We will remedy this situation, in the next section, by providing a fully worked out example.

\section{THE FUNDAMENTAL PROBLEM OF THE TWO-FLUID APPROXIMATION: AN ILLUSTRATION}

Let us begin this section by briefly outlining what is meant by neighbouring word lines, in the context of single-fluid approximation in cosmology. This preamble is necessitated by the need for clarity in discussing the differences between the single-fluid approximation and the multifluid approximation treatment that will be later developed. Let $X^{\mu}$ be a vector whose components are given in a comoving coordinate by $X^{\mu}=\delta x^{\mu}\left(X^{0}=0\right)$, which at all times joins the two world lines given by $x^{\mu}$ and $x^{\mu}+\delta x^{\mu}$. The reader is referred to Figure 1 for a schematic representation of this set-up. Since this is a comoving sys-

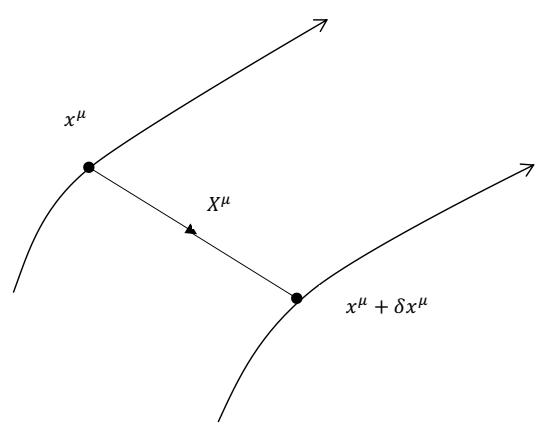

FIGURE 1: Comoving observers and a connecting vector.

tem, there is one fundamental 4 -velocity vector. The connector $X^{\mu}=\left(\partial x^{\mu} / \partial x^{v}\right) \delta x^{v}$ does not lie in the rest frame defined by the 4 -velocity. However, it is possible to define the time derivative and a relative position vector using the velocity vector and a projection tensor that is projected onto the rest frame of such a velocity; i.e.,

$$
\begin{aligned}
& \dot{X}^{\mu}=u_{; \nu}^{\mu} X^{v}, \\
& X_{\perp}^{\mu}=h^{\mu}{ }_{v} X^{v} .
\end{aligned}
$$

It follows that there exists a corresponding relative velocity vector $V^{\alpha}=V^{\alpha}{ }_{\beta} X_{\perp}^{\beta}$, where $V^{\alpha}{ }_{\beta}=h^{\alpha}{ }_{\gamma} h_{\beta}^{\delta} u_{; \delta}^{\gamma}$. This indicates that the relative velocity vector of the neighbouring comoving particles is linked to the relative position vector through a linear transformation as given by the spatial gradient of the 4 -velocity vector. The relative vector can then be covariantly split into an expansion parameter and a vorticity parameter as is done, for example, in the $3+1$ covariant formulation of Einstein field equations [29, 30, 31,32]. We emphasise that this presentation is for a comoving velocity and hence single-fluid approximation. What if the two neighbouring observers are not comoving? Could we formulate projection tensors related to the two velocities and what could we learn from this? It is clear that time parameters would be different ()$^{\cdot}=w^{\mu} \tilde{\nabla}_{\mu}()$ and ()$^{\prime}=v^{\mu} \nabla_{\mu}()$, assuming two different velocity vectors $w^{\mu}\left(\equiv u_{\mid X}^{\mu}\right)$ and $v^{\mu}\left(\equiv u_{\mid Y}^{\mu}\right)$. The two spacial derivatives are covariant (along the surfaces as described by the metrics $\left.g_{\mu v}\right|_{w}$ and $g_{\mu \nu}$, respectively). One could theorise about possible projection tensors $h_{\mu v_{\mid w}}=f\left(\left.g_{\mu v}\right|_{w}, w_{\mu}, w_{v}\right), h_{\mu v_{\mid v}}=f\left(g_{\mu v}, v_{\mu}, v_{v}\right)$, and the intriguing case $h_{\mu v_{\mid w v}}=f\left(\left.g_{\mu v}\right|_{\mid w, v}, w_{\mu}, v_{v}\right)$ (the reader is referred to the notes [33] for a detailed discussion on projection tensors). The projectors $h_{\mu v_{\mid w}}$ and $h_{\mu v_{\mid v}}$ are the familiar tensors found in the literature. $h_{\mu v_{\mid w v}}$ is new and demands further investigation. The fundamental problem in this case is how the two velocities couple to give rise to an effective velocity. There are many different possible configurations that could yield such effective velocity, for example, the configuration of noninteracting fluids considered in [34] where anisotropy was studied. In this article, we will present an alternative configuration, as an illustration.

Let $w_{\mu}$ and $v_{\mu}$ be two 4 -velocity unit vectors $\left(w^{\mu} w_{\mu}=-1=\right.$ $v^{\mu} v_{\mu}$ ) that give rise to a complex 4 -velocity $u_{\mu}$ defined by

$$
u_{\mu}=w_{\mu}+i v_{\mu}
$$

and whose conjugate is

$$
u^{\mu}=w^{\mu}-i v^{\mu},
$$

where $i=\sqrt{-1}$. This can be found by requiring the existence of Cauchy-Riemann-like equations for the 4-dimensional objects, from which a complex potential $\Phi(w, v)[41]$ may be defined. A complementary scalar potential function was used in formulating the dissipative relativistic fluid theory of the divergence type; the reader is referred to the appendix (Appendix A) for a summary. In our case, we need $u^{\mu}$ to be analytic with respect to a covariant derivative at a given event [42]. It is then clear from Equations (17) and (18) that

$$
u^{\mu} u_{\mu}=w^{\mu} w_{\mu}+v^{\mu} v_{\mu}=-2,
$$

from which we can define the fundamental relation

$$
\frac{u^{\mu}}{\sqrt{2}} \frac{u_{\mu}}{\sqrt{2}}=-1
$$

We see in this section that two unit vectors in a complex configuration generates a unit vector $\hat{u}=u / \sqrt{2}$. We can now investigate the implication of having such a complex 4-velocity vector. Some work on this kind of modelling has been done, for example, in $[35,36,37]$.

\subsection{Effective Velocity for More Fluid Species}

An important question to address before we continue is how one constructs the effective 4-velocity given more velocity fields. For example, let $w, v, \xi$, and $\zeta$ be 4 -velocities in a complex configuration. Three of these give rise to an effective 4 -velocity as follows:

$$
u_{\mu}=w_{\mu}+i\left(v_{\mu}+i \xi_{\mu}\right)=\left(w_{\mu}-\xi_{\mu}\right)+i v_{\mu} .
$$

The case of four components is given by

$$
u_{\mu}=w_{\mu}+i\left[v_{\mu}+i\left(\xi_{\mu}+i \zeta\right)\right]=\left(w_{\mu}-\xi_{\mu}\right)+i\left(v_{\mu}+\zeta\right) .
$$

The extension to a greater number of velocity fields in this formalism should be straight forward. We will restrict our discussion to the case of two fluid species. 


\subsection{A projection Tensor}

We begin by constructing a projection tensor onto an emergent surface using the 4-velocity $\hat{u}^{\mu}$ (where we define $\hat{u}^{\mu}=u^{\mu} / \sqrt{2}$ ). Such a projection tensor will take the generic form

$$
\hat{h}_{v}^{\mu}=\hat{g}^{\mu}{ }_{v}+\hat{u}^{\mu} \hat{u}_{v}
$$

and is defined to obey the orthogonality condition $\hat{h}_{v}^{\mu} \hat{u}^{\mu}=0$. The associated projected tensor $h^{\mu}{ }_{v}=g^{\mu}{ }_{v}+w^{\mu} w_{v}$ is projected onto the rest frame only if there is no vorticity. For example, the projection tensor used in Friedmann-Walker models with perfect fluids matter is characterised by local isotropy [28]. But the tensor $\hat{h}_{v}^{\mu}$ is not the same as $h^{\mu}{ }_{v}$ and is not projected onto the hyper-surfaces defined by either $w_{\mu}$ or $v_{\mu}$ but rather to one defined by $\hat{u}^{\mu}$. It is easy to show that if $w_{\mu} \equiv v_{\mu}$, then

$$
u^{v} u_{v}=v^{v} v_{v}(1+i)(1-i)=2 v^{v} v_{v}=-2,
$$

recovering the result in Equation (19). We now have a projection tensor $\hat{h}=\hat{h}\left(g_{\mu v}, \hat{w}_{\mu}, \hat{v}_{v}\right)$ which can be resolved into the fundamental velocities $w_{\mu}$ and $v_{\nu}$.

$$
\begin{aligned}
\hat{h}^{\mu v} & =g^{\mu v}+\hat{u}^{\mu} \hat{u}^{v}=g^{\mu v}+\frac{1}{2} u^{\mu} u^{v} \\
& =g^{\mu v}+\frac{1}{2}\left[\left(w^{\mu} w^{v}-v^{\mu} v^{v}\right)+i\left(v^{\mu} w^{v}+v^{v} w^{\mu}\right)\right] \\
& =\frac{1}{2}\left[g^{\mu v}+\left(w^{\mu} w^{v}-v^{\mu} v^{v}\right)+g^{\mu v}+i\left(v^{\mu} w^{v}+v^{v} w^{\mu}\right)\right] \\
& =\frac{1}{2}\left[g^{\mu v}+\left(w^{\mu} w^{v}-v^{\mu} v^{v}\right)+i\left(-i g^{\mu v}+v^{\mu} w^{v}+v^{v} w^{\mu}\right)\right] \\
& =\hat{h}_{\mu v(R)}+i \hat{h}_{\mu v(C)},
\end{aligned}
$$

where $R$ denotes the real part, while $C$ denotes the complex part.

The presentation above looks deceptively familiar but the projection tensor separates velocities that do not have same velocity pointing in different directions as is standard in the literature. This distinction should always be kept. We can now ask the question, how does this affect the energy momentum tensor in the case of the general relativistic multifluid? We will restrict our discussions to the case of two relativistic fluids. Here, we employ nonstandard decomposition as will be explained.

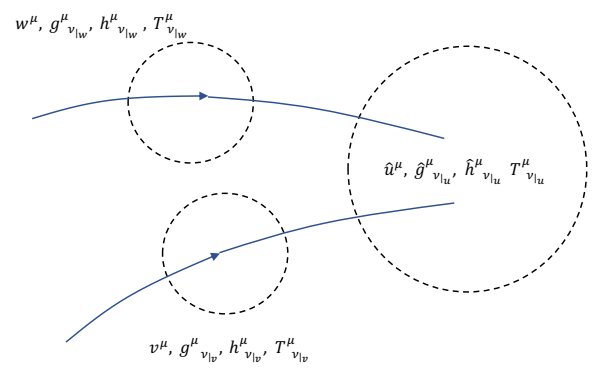

FIGURE 2: Two original separate velocity vectors, projection tensors, and energy momentum tensors and the resultant.

The substructure of the projection tensor induced by the different 4-velocities affects the fundamental equations for the multifluid system, which in turn affects the momentum, energy, and mass equations. In order to grasp the consequences of such substructuring, we first consider heat conduction. Rather than following the standard bottom-up approach where the equation representing the bulk behaviour is derived from subconstituents states, we adopt a top-down approach. In this case, we decompose the equations for the bulk into the constituent parts. Let's begin with the heat vector whose divergence contributes to the energy balance equation.

\subsection{The Heat Flux Vector}

Using the projector tensor $\hat{h}_{\mu \nu}$, we can define a heat vector with respect to the velocity $\hat{u}_{\gamma}$. We find

$$
\begin{aligned}
\hat{q}_{\mu}= & -\hat{h}_{\mu \nu} \hat{u}_{\gamma} T^{v \gamma} \equiv-\hat{h}_{\mu \nu}\left(\frac{u_{\gamma}}{\sqrt{2}}\right) T^{v \gamma}, \\
= & -\frac{1}{\sqrt{2}}\left(\hat{h}_{\mu \nu(R)}+i \hat{h}_{\mu \nu(C)}\right)\left(w_{\gamma}+i v_{\gamma}\right) T^{v \gamma}, \\
= & -\frac{1}{\sqrt{2}}\left[\left(\hat{h}_{\mu \nu(R)} w_{\gamma}-\hat{h}_{\mu \nu(C)} v_{\gamma}\right)\right] T^{v \gamma} \\
& -\frac{1}{\sqrt{2}} i\left[\left(\hat{h}_{\mu \nu(C)} w_{\gamma}+\hat{h}_{\mu \nu(R)} v_{\gamma}\right)\right] T^{v \gamma} .
\end{aligned}
$$

This shows that the heat vector is composed of real and complex parts. It follows that heat vector's contribution to the stress momentum tensor is given by

$$
\hat{q}_{(\mu} \hat{u}_{v)}=-\hat{h}_{(\mu \gamma} \hat{u}_{\delta} T^{\gamma \delta} \hat{u}_{v)}=-\frac{1}{\sqrt{2}}\left(\hat{h}_{(\mu \nu} \hat{u}_{\delta}\right) T^{v \delta}\left(w_{v)}+i v_{v)}\right),
$$

where each term with a hat, $\hat{f}$, can be decomposed into real and complex parts, respectively. Equations (25) and (26), together, yield

$$
\begin{aligned}
\hat{q}_{(\mu} \hat{u}_{v)}= & \hat{h}_{(\mu \gamma} \hat{u}_{\delta} T^{\gamma \delta} \hat{u}_{v)} \\
= & \frac{1}{2}\left[\left(\hat{h}_{\mu \nu(R)} w_{\delta} w_{v)}-\hat{h}_{\mu \nu(C)} v_{\delta} w_{v)}\right)\right] T^{v \delta} \\
& -\frac{1}{2}\left[\left(\hat{h}_{\mu \nu(C)} w_{\delta} v_{v)}+\hat{h}_{\mu \nu(R)} v_{\delta} v_{v)}\right)\right] T^{v \delta} \\
& +\frac{1}{2} i\left[\left(\hat{h}_{\mu v(R)} w_{\delta} v_{v}-\hat{h}_{\mu v(C)} w_{v} v_{\delta}\right)\right] T^{v \delta} \\
& +\frac{1}{2} i\left[\left(\hat{h}_{\mu v(C)} w_{\delta} w_{v)}+\hat{h}_{\mu v(R)} v_{\delta} w_{v)}\right)\right] T^{v \delta} .
\end{aligned}
$$

\subsection{Energy Density and Pressure Terms}

The energy density is constituted as follows:

$$
\hat{\rho}=\hat{u}_{\mu} \hat{u}_{v} T^{\mu v} \equiv \frac{1}{2}\left(w_{\mu} w_{v}-v_{\mu} v_{v}\right) T^{\mu v}+\frac{i}{2}\left(w_{\mu} v_{v}+w_{\mu} v_{v}\right) T^{\mu v} .
$$

Likewise, the isotropic pressure term is given by

$$
\hat{p}=\frac{1}{3} \hat{h}_{\mu \nu} T^{\mu v}=\frac{1}{3}\left(\hat{h}_{\mu v(R)}+i \hat{h}_{\mu \nu(C)}\right) T^{\mu \nu} .
$$

The anisotropic pressure is given by

$$
\begin{aligned}
\hat{\pi}_{\mu \nu}= & T^{\gamma \delta}\left(\hat{h}_{\gamma\langle\mu(R)} \hat{h}_{v\rangle \delta(R)}-\hat{h}_{\gamma\langle\mu(C)} \hat{h}_{v\rangle \delta(C)}\right) \\
& +i T^{\gamma \delta}\left[\left(\hat{h}_{\gamma\langle\mu(R)} \hat{h}_{v\rangle \delta(C)}\right)+\left(\hat{h}_{\gamma\langle\mu(C)} \hat{h}_{v\rangle \delta(R)}\right)\right],
\end{aligned}
$$

where the real and complex parts are clearly manifest. 


\subsection{The Energy Momentum Tensor}

The total stress-energy momentum tensor takes the form

$$
T_{\mu v}=\hat{\rho} \hat{u}_{\mu} \hat{u}_{v}+\hat{p} \hat{h}_{\mu v}+2 \hat{u}_{(\mu} \hat{q}_{v)}+\hat{\pi}_{\mu v}
$$

which structurally resembles the standard single-fluid form but hides the real and complex constituents. An observer living in the real plane, with no knowledge of the existence of the complex plane, will measure a total energy density that does not match what they expect, i.e., $\left(w_{a} w_{b}-v_{a} v_{b}\right) T^{a b} / 2$ as seen in Equation (27) instead of $w_{a} w_{b} T^{a b}$ given by the own 4-velocity $w^{a}$. This disparity between the expected and the observed measures may help account for some of the disparities between predictions from single-fluid approximation and what is observed in cosmology. In general, this form of flow is anisotropic and may provide a test-ground for the cosmological principle. In our case, we have considered the limit in which the system is isotropic.

The illustrative velocity of $\hat{u}_{c}$ is but one example of how two fluids could be coupled. It belongs to a family of couplings that are expressed as $\hat{u}_{C}=f\left(w_{C}, v_{C}, \ldots\right)$ where ${ }^{\prime} .{ }^{\prime}$ expresses the fact that there may be more velocity fields. We know that other configurations [34, 38, 39] have been used to study anisotropic models where the energy-stress tensor is primarily mattered tensors. In this article, we consider such couplings in the context of thermodynamics.

\subsection{The Entropy Vector}

There are two formal definitions of entropy: the thermodynamic and the statistical. We take the thermodynamic viewpoint. In this regard, the classical thermodynamics theory considers a system that is composed of constituents, but whose state is found by taking the averages of thermodynamic properties of such constituent, in effect, looking at the cumulative behaviour. Although the initial development of the concept only considered such averages for a system that was in equilibrium via statistical mechanics, the latter development extends the theory by incorporating aspects that allowed for the nonequilibrium thermodynamics via the kinetic theory. It is the latter version that is of interest to us. Standard treatment of statistical thermodynamics, see [40], is based on postulates that are given in terms of the behaviour of simple systems. These are systems microscopically homogeneous, isotropic, and devoid of electric charge, chemical reactions, electrical force fields, or surface effects. In order to account for multifluids, where some of these properties cannot be neglected, it is imperative that we go beyond the simple system postulates. To this end, we follow $[1,2,3]$ who, by incorporating the quadratic terms in the heat flux and viscous stresses in the expression for the entropy 4-vector, obtain a generalised theory able to describe transient nonequilibrium thermodynamics satisfying the causality condition. It is straightforward to show that the entropy current for this flow takes the following form:

$$
\begin{aligned}
S_{\mid i}^{\mu}= & \hat{s} \hat{u}_{\mid i}^{\mu}+\hat{s} \mu_{\mid i} \\
\equiv & \hat{s} \hat{u}_{\mid i}^{\mu}+\hat{q}_{\mid i}^{\mu} \frac{1}{T}-\left(\hat{\beta}_{0} \Pi^{2}+\hat{\beta}_{1} \hat{q}_{v \mid i} \hat{q}_{\mid i}^{v}+\hat{\beta}_{2} \hat{\pi}_{\gamma \delta \mid i} \hat{\pi}^{\gamma \delta \mid i}\right) \frac{\hat{u}_{\mid i}^{\mu}}{2 T} \\
& +\left(\hat{\alpha}_{0} \Pi \hat{q}_{\mid i}^{\mu}+\hat{\alpha}_{1} \hat{\pi}_{\mu v \mid i} \hat{q}_{\mid i}^{v}\right) \frac{1}{T} .
\end{aligned}
$$

Here too, any term with a hat, $\hat{f}$, can be expanded in terms of a real part and an imaginary part. $\hat{s}$ is the entropy density, and $\hat{s}^{\mu}$ is the entropy flux with respect to $\hat{u}^{\mu \mid i}$ such that $\hat{s}_{\mu \mid i} \hat{u}_{\mid i}^{\mu}=0$. $\Pi$ is the bulk viscosity. Here, we have considered the limit in which the temperature is universal $[7,23]$. The coefficients $\hat{\beta}_{0}$, $\hat{\beta}_{1}, \hat{\beta}_{2}, \hat{\alpha}_{0}$, and $\hat{\alpha}_{1}$ are the generalized case of the counterparts appearing in the MIS theory.

\section{DISCUSSION AND CONCLUSION}

We have developed a generic expression for stress-energymomentum tensor and entropy taking into account a multifluid configuration. The formulation extends the MIS theory by incorporating more than one fluid species. Our starting point is the construction of the effective 4-velocity $\hat{u}^{\mu}$ that is the resultant of the various fluid species velocities and which is defined by the Cauchy-Riemann equations for 4 dimensions [41]. The standard approach in modelling nonconducting fluid species uses the velocity of the centre of mass or gravity as the representative velocity and the whole fluid is then treated as a single fluid. In contrast, we use a complex formulation which allows us to retain and monitor the unique or peculiar contributions from individual species. This allows for the treatment of bulk behaviour [11] and that incorporates fluid interactions that may or may not be expressed by Equation (12). This is particularly important for the treatment of well-behaved heat conduction in relativistic fluids that includes dissipation $[44,26]$.

So what is the utility of this formulation? It is thought that the recently discovered late time acceleration of the universe could be explained by invoking dark energy whose density is usually added into the Friedman equation by hand, without a hint of its source. We think that this can be remedied. In the illustration, we have considered a formulation that brings two fluids together whose effective 4-velocity can be expressed as a complex vector. This plays two roles: (i) it ensures that the fluids remain distinct and (ii) it allows for two fluids to have an impact on the other. From an observer's point of view, the one living in the real part will not have any knowledge of the existence of the complex dynamics but will notice that the total energy density is not what they expect, i.e., $\rho_{\left.\right|_{X}}=w_{\mu} w_{\nu} T^{\mu \nu}$, but one that is modulated by some mysterious addition $\left(w_{\mu} w_{v}-v_{\mu} v_{v}\right) T^{\mu v} / 2 \equiv \rho_{\left.\right|_{X}}+\rho_{\left.\right|_{Y}}$. The negative sign here is an artefact of the complex analysis not necessarily bearing direct physical significance. The observer in the imaginary hypersurface will, similarly, notice the difference in their energy density. To see the impact of this, consider recent observations that strongly suggest the existence of cosmic acceleration. It follows that the cumulative effect of all the species in the real frame, for two or more fluid models, could help explain $\ddot{a}>0$ leading to Friedmann acceleration equation

$$
\frac{\ddot{a}}{a}=-\frac{1}{6} \sum_{i}\left(\rho_{i}+3 p_{i}\right),
$$

where $8 \pi G=1=c^{2}$.

This hints at a twin-universe theory and by extension a multiverse theory. Since there are numerous ways of formulating an effective 4-velocity, it is clear that our formulation belongs to such a family and demands further investigation. 


\section{ACKNOWLEDGEMENT}

The author acknowledges the funding support from the University of Cape Town's Next Generation Professoriate Programme (NGP).

\section{Appendix A. A GENERIC APPROACH: THE DISSIPATIVE RELATIVISTIC FLUID THEORIES OF DIVER- GENCE TYPE}

Let the label $X$ denote a relativistic dissipative fluid moving with a 4 -velocity $w^{c}[26,43]$. We have decided to keep the index $X$ as a reminder that we have a single fluid that is identified by this label. Let the fluid satisfy the following properties:

a) The dynamical variables are the individual particlenumber current vectors, $N_{X}^{\mu}$, and the total stress-energy momentum tensor $T_{X}^{\mu \nu}$.

b) The conservation laws in addition to the dynamical equations are

$$
\begin{aligned}
\nabla_{\mu} N_{X}^{\mu} & =0, \\
\nabla_{\mu} T_{X}^{v \mu} & =0, \\
\nabla_{\mu} A_{X}^{\mu \nu \delta}-I_{X}^{v \delta} & =0,
\end{aligned}
$$

where the covariant derivative based on a space-time geometry endows with the metric tensor $g^{\mu \nu}$. It is known [33] that covariant derivatives on space-time and the covariant derivatives defined in the geometry of a spacelike hyper-surface in space-time are linked through the projection of a space-like connection. $A$ and $I$ are the algebraic forms of symmetric and the trace-free $N_{X}^{v}$ and $T^{v \mu}$.

c) There exists a combined entropy current $s_{X}^{v}$ that satisfies

$$
\nabla_{v} s_{X}^{v}=\sigma,
$$

where $\sigma$ is some algebraic form of $N_{X}^{v}$ and $T_{X}^{v \mu}$. Reversible or thermodynamic equilibrium state is given when $\sigma=0$, while the irreversible state is when $\sigma>0$.

Theories that satisfy these conditions are called divergence theories.

$$
h_{\mid X}^{\mu v}=g_{\mid X}^{\mu v}+u_{\mid X}^{\mu} u_{\mid X}^{v} .
$$

It is important to note that the resultant velocity $u_{\mid X}^{v}$ is a generic vector function whose form is dependent on the velocities of the two fluid species; an example was considered in the previous section.

The reader will note that the presentation above extends those of [5] to include two coupled particle types. Indeed, this can be generalised to include more particle species. In the twospecies case, the general theory obeying the three properties above may be shown to be generated by the scalar potential $\chi_{X}$ and a tensor $I_{X}^{v \mu}$

$$
\begin{aligned}
N_{X}^{\mu} & =\frac{\partial^{2} \chi_{X}}{\partial \xi^{2} \xi_{\mu}} \\
T_{X}^{\mu \nu} & =\frac{\partial^{2} \chi_{X}}{\partial \xi_{\mu} \partial \xi_{v}}, \\
A_{X}^{v \mu \gamma} & =\frac{\partial^{2} \chi_{X}}{\partial \xi_{\mu} \partial \xi_{v \gamma}}
\end{aligned}
$$

with the entropy current given by

$$
\begin{aligned}
S^{v} & =\left(\frac{\partial \chi_{X}}{\partial \xi_{v}}-\xi N_{X}^{v}\right)-\xi_{\mu} T_{X}^{v \mu}-\xi_{\mu \gamma} A_{X}^{v \mu \gamma}, \\
\sigma & =-\xi_{v \mu} I^{v \mu} .
\end{aligned}
$$

Equation (A.10) is the result of taking the divergence of equation (A.9) given the three properties (Appendix A). It is straight forward to show that this is a general theory satisfying these properties. The velocity fields are chosen in such a way that the entropy density satisfies the concavity requirement [3]. The selection is nontrivial in general relativity since the definition of entropy density is frame-dependent, which in itself is not unique. In order to make progress, we assume that privileged frames, satisfying the above requirements, exist [4]. In this regard, the stress-energy momentum tensor may be written in the form

$$
\begin{aligned}
& T_{X}^{\mu v}=\left(\rho_{X} u^{\mu} u^{v}+p_{X} h^{\mu v}+2 u_{X}^{(\mu} q_{X}^{v)}+\pi_{X}^{\mu v}\right), \\
& N_{X}^{\mu}=n_{X} u_{X}^{\mu}+n_{X}^{\mu},
\end{aligned}
$$

such that $n_{X}^{v} u_{X v}=0$. The description is generic but can easily be adapted to recover some of the known theories. For example, Eckart's theory is easily recovered when one restricts the above formulation to the first-order approximation and the MIS theory in the previous section for the second-order approximation (see [5]). These formulations are useful for modelling causally well-behaved heat conduction in relativistic fluids $[44,26]$ in the context of single-fluid approximation. The approach above is based on defining a scalar type generating function that gives rise to the fundamental vector and tensor dynamical variables. These parameters are locally defined and allow for the notion of a thermodynamic equilibrium. The technique is not dissimilar to the decomposition of cosmological perturbations into local scalar type, local vector type, and local tensor type [48]. This is in contrast to the standard Helmholtz's theorem used to define nonlocal scalars and vectors [45, 46, 47]. The challenge with nonlocal variables is that one needs to specify boundary conditions in order to define such variables. Although it is often difficult to map theories derived from locally defined variables to those not based on nonlocal variables, it is possible to find a set that allows for such links. For example, it is possible to reconstruct a nonlocal theory, using divergence derivatives of a scalar variable, into a corresponding local theory as demonstrated in [48]. In this regard, the scalar variable, or better still a function, becomes a generator of the specified field theory.

\section{References}

[1] W. Israel, Ann. of Phys. 100(1976) 310.

[2] W. Israel and J.M. Stewart, Ann. Phys. 118(1979)341.

[3] I. Müller, Speeds of Propagation in Classical and Relativistic Extended thermodynamics, Living (1999).

[4] T. Ruggeri T (1990), Continuum Mech. Thermodyn.2 (1992) 4.

[5] R. Geroch and L. Lindblom, Phys. Rev. D 41(1990) 6.

[6] D. Priou, Phys. Rev. D 43 (1991) 4.

[7] B. Osano and T. Oreta, preprint arXiv:1904.08670.

[8] P. T. Landsberg and G. E. A. Matsas, Phys. Lett. A223(1996) 401. 
[9] P. T. Landsberg and G. E. A. Matsas, Phys. Lett. A340(2004) 92.

[10] B. Osano B, Fundamental Thermodynamics Relation: A perturbation approach, in preparation (2020).

[11] T. Padmanabhan, AIP Conf. Proc. (2010) 1241.

[12] T. K. Nakamura, Phys. Lett.A(2006).

[13] C. Eckart, Phys. Rev.58(1940) 919.

[14] G. A. Kluttenberg, S. R. de Groot and P. Mazur, Physica,19(1953)689.

[15] G. A. Kluttenberg, S. R. de Groot and P. Mazur, Physica,21(1955) 148.

[16] L. Landau and E. M. Lifshitz, in Fluid Mechanics (Addison Wesley, Reading, Mass. 1958).

[17] C. Cattaneo, C. R. Acad. Sci (Paris)247 (1958)431.

[18] M. Kranyz, Nuovo Cimento 42B (1966) 51.

[19] D. M. Aitken, Relativistic Irreversible Thermodynamics, M.Sc. Thesis, University of Alberta, Edmonton, 1967.

[20] N. G. van Kampen, Physica 46 (1970) 315.

[21] J. Stewart, in Lecture notes in Physics( Springer, Berlin,1971).

[22] J. F. Salazar and T. Zannias, eprint arXiv:1904.04368.

[23] B. Osano, Universal temperature in preparation (2020).

[24] N-T. Nguyen, in Micro mixers - Fundamentals, Design and Fabrication, (William Andrew Young Publisher, 2008).

[25] D. Jou et al 1988 , Rep. Prog. Phys. ,51 1105.

[26] N. Andersson and G. L. Comer Living Reviews in Relativity 10(2007) 1.

[27] R. H. Gowdy (1994), J. Math. Phys. 35, 1274.

[28] G. F. R. Ellis and H. Van Elst (1999), Cosmological Models, NATO Adv. Study Inst. Ser. C. Math. Phys. Sci. 541,1.

[29] J. Ehlers, Gen. Rel. Grav.25(1993)1225.

[30] G. F. R. Ellis, in Cargèse Lectures in Physics Vol. 6, Ed. E Schatzman (Gordon and Breach, New York, 1973).

[31] G.F.R. Ellis and H. van Elst, NATO Sci. Ser. C 541 (1999) 1.

[32] B. Osano, Class. Quant. Grav. 34 (2017)125004.

[33] R. Gowdy, http:// www.people.vcu.edu/ rgowdy/phys691/pdf, accessed in November 2019.

[34] S.S. Bayin, Astrophy. J 303(1986).

[35] W. Zimdahl, H. Velten, W. Algoner, Matter Growth in Imperfect Fluid Cosmology, preprint arXiv: 1903.03383.

[36] S. Carneiro et al, Unphysical properties in a class of interacting dark energy models, preprint arXiv: 1909.10336

[37] W. Zimdahl et al, On (non-)dynamical dark energy, preprint arXiv: 1911.12084.

[38] S.S Bayin,Phys. Rev. D26 (1982) 1262.

[39] P. S. Letelier, Phys. Rev. D 22 (1980) 807.

[40] C. L. Tien and J. H. Lienhard, in Statistical Thermodynamics, (Hemisphere Publishing Corporation, 1979)

[41] B. Osano, Cauchy-Riemann equations in higher dimensions, in preparation 2020.

[42] Here the variables are 4-dimensional, with one dimension giving the temporal and the reaming three the spatial. We therefore deal with events rather than points in our development.

[43] L. Rezzolla L and O. Zanotti Relativistic Hydrodynamics (Oxford University Press.) (2013)

[44] B. Carter in Relativistic Fluid Dynamics of Lecture Notes in Mathematics (eds A. Anile, and M. Choquet-Bruhat, Springer, 1989).

[45] J. M. Stewart and M. Walker, Proc. Roy. Soc. Lond. A341 (1974) 49.
[46] H. Kodama and M. Sasaki, Prog. Theor. Phys. Suppl. 78(1984) 1.

[47] J. M. Stewart, Class. Quant. Grav.7 (1990) 1169.

[48] C. Clarkson C and B. Osano B, Class. Quant. Grav. 28 (2011) 22. 